\title{
Tecnologías de la información y comunicación como estrategia para fortalecer la competencia Interpretativa y productiva de textos ${ }^{1}$
}

Information and communication technologies as strategies to
strengthen the Interpretation and production of texts

DOI: http://dx.doi.org/10.17981/cultedusoc.9.3.2018.74

Artículo de investigación. Fecha de recepción: 15/06/2018. Fecha de aceptación: 27/11/2018

Luaura Chávez-Obregón²;

Lívida Escorcia-Rudas; Alva Montenegro-De Pertuz; Yolanda Cantillo-Molina;

Celina Pabón-Pallares; María Gonzales-Castañeda; Edgardo Villa Real-Martínez; Javier Guerrero-Guerrero; Alfonso Lobato-Petuz; Heber López-Pérez;

Olfa Cantillo-Bolaño; Arquímedes Hernández-Ariza; Adelso Gómez-Hernández;

Lucila Polo-Sánchez; Clementina Villamil; Arelys Villa-Páez;

Emerson Pertuz-Samper; Lila De La Hoz-Cera; José Sánchez-Paz;

Hernando Vega-Cantillo; Rosmari Mozo-Carranza; Andrés Villafañe-Ayala;

Yosmar Ropain-Caballero; María De la Cruz; Omar Potes-Donado;

Rosmira Miranda-Sánchez y Luxer Serrano-Cantillo ${ }^{3}$

Institución educativa Departamental Agropecuaria Nuestra Señora de las Mercedes (Colombia) laurmil28@hotmail.com

Para citar este artículo:

Chávez-Obregón, L., Escorcia-Rudas, L., Montenegro-De Pertuz, A., Cantillo-Molina, Y., Pabón-Pallares, C., Gonzales-Castañeda, M., Villa Real-Martínez, E. Guerrero-Guerrero, J., Lobato-Petuz, A., López-Pérez, H., Cantillo-Bolaño, O., Hernández-Ariza, A., GómezHernández, A., Polo-Sánchez, L., Villamil, C., Villa-Páez, A., Pertuz-Samper, E., De La Hoz-Cera, L. Sánchez-Paz, J., Vega-Cantillo, H., Mozo-Carranza, R., Villafañe-Ayala, A., Ropain-Caballero, Y., De la Cruz, M., Potes-Donado, O., Miranda-Sánchez, R. y Serrano-Cantillo, L. (2018). Tecnologías de la información y comunicación como estrategia para fortalecer la competencia Interpretativa y productiva de textos. Cultura. Educación y Sociedad 9(3), 627-634. DOI: http://dx.doi.org/10.17981/cultedusoc.9.3.2018.74

\section{Resumen}

La interpretación y producción de textos siempre ha sido una temática de gran interés investigativo a nivel nacional, debido a los problemas vinculados con los bajos resultados obtenidos en las diferentes pruebas establecidas. Partiendo de esto el propósito de la investigación está dirigido a fortalecer en los estudiantes la competencia Interpretativa y productiva de textos a través de las Tecnologías de la información y comunicación. Se asume un abordaje metodológico desde la investigación cualitativa, orientada desde la investigación acción. La unidad de análisis estuvo constituida por 132 estudiantes de los grados 4 y 5 , entre las edades de 0 a 19 años, que conforman las diferentes sedes de la IED Agropecuaria Nuestra Señora de las Mercedes. Los resultados arrojados dejaron ene videncia que al trabajar los temas referentes a la interpretación y producción de textos, se generó un incremento significado en los resultados académicos de los estudiantes; entregando productos de calidad en contenido e interpretación textual.

Palabras clave: Interpretación, producción, tecnologías de la información y la comunicación

\section{Abstract}

The interpretation and production of texts has always been a topic of great research interest at the national level, due to the problems associated with the low results obtained in the different tests established. Based on this, the purpose of the research is aimed at strengthening students' interpretive and productive competence in texts through information and communication technologies. It assumes a methodological approach from qualitative research, oriented from the action research. The analysis unit consisted of 132 students of grades 4 and 5 , between the ages of 0 to 19 years, which make up the different venues of the IED Farming Our Lady of Mercy. The results thrown away showed that by working on issues related to the interpretation and production of texts, a significant increase in the students' academic results was generated; delivering quality products in content and textual interpretation.

Keywords: Improvement in the interpretation and production of texts in students, curricular integration of the IEP supported in ICT.

1 Este artículo ha sido derivado del Programa de Fortalecimiento de la Cultura Ciudadana y Democrática CT+I a través de la IEP apoyada en TIC en el Departamento de Magdalena: proyecto Ciclón

2 Líder del Grupo de investigación "Los Socráticos".

3 Docentes de la IED Agropecuaria Nuestra Señora de las Mercedes, del grupo de investigación "Los socráticos".

- The author; licensee Universidad de la Costa - CUC

Cultura, Educación y Sociedad vol. 9 no. 3, pp. 627-634. Diciembre, 2018

Barranquilla. ISSN 2389-7724 Online 


\section{Introducción}

Las dificultades presente en los estudiantes a nivel académico en cuanto a la interpretación y producción de textos en las instituciones educativas, ha sido un tema tratado constantemente a nivel nacional; debido a que los resultados obtenidos en las pruebas internacionales que evalúan el componente de lectura, siguen arrojando resultados desalentadores para Colombia. Lo anterior evidencia que los estudiantes que presentan las pruebas, no tienen la capacidad de interpretar y comprender los contenidos de información expuestos en las mimas (Barletta, Toloza, Villar, Rodríguez, Bovea y Moreno, 2013).

Debido a esos resultados arrojados en las pruebas tanto nacionales como internaciones el Instituto Colombiano para el fomento de la educación superior (ICFES), diseño e incorporo nuevos elementos evaluativos en lo referente a las pruebas aplicadas SABER; cuyo objetivo radica en evaluar en términos de resultados las capacidades de los estudiantes en las áreas específicas de lenguaje, matemática, ciencias, ambiente y sociedad. En cuanto al área de lenguaje, se valoran las competencias comunicativas entre ellas la competencia lectora e interpretativa. (ICFES, 2015).

Estos resultados no se alejan de la realidad que se vive en las instituciones educativas a nivel departamental, donde se presenta al departamento del Magdalena con pocos cambios significativos en el índice sintético de la calidad 2015, en cuanto al tema de comprensión lectora, donde se mostró un incremento en esta competencia en el nivel de básica primaria, pero sigue presentando falencias en la secundaria. Estos datos coinciden con los resultados de la Institución Educativa Departamental Agropecuaria Nuestra Señora de las Mercedes, la cual se encuentra constituida por estudiantes de la básica primaria y media distribuida entre diferentes sedes de edu- cación; quienes presentan un rendimiento relativamente homogenizada en cuanto al rendimiento académico se refiere, evidenciándose en las pruebas SABER realizadas por el ICFES para el periodo 2016, donde más del $60 \%$ de la población evaluada entre los grados $3^{\circ} 5^{\circ}$ y $9^{\circ}$ presentan puntuaciones entre mínimas e insuficientes para la competencia de lenguaje; como también las distintas áreas del conocimiento evaluadas por el ICFES 2015; esto induce al cuerpo docente, a relacionar estos puntajes con los resultados académicos a nivel interno, a relacionar dichas dificultades con la falencia en competencias de interpretación y producción de textos en los estudiantes, lo que está afectando de manera general el proceso de formación y por ende la calidad educativa de la institución.

Es necesario reconocer que los modelos pedagógicos que aún se imparten en la escuela obedecen a una pedagogía tradicional; donde se presentan las clases de manera magistral, con procesos pasivos y poca participación; problemática que está en proceso de solución; pero que requiere la adaptación de nuevas estrategias metodológicas que impliquen los nuevos elementos conceptuales que ofrece la ciencia y la tecnología moderna (Martínez, Salgado, Cantillo y Prada 2015). Esta solución entonces requiere de un proceso de innovación curricular, de la implementación de un nuevo método de enseñanza que pueda tener pertinencia desde la integración curricular de las tecnologías de la información y la comunicación, en el contexto escolar; en la cual se permitan abordar desde un proceso reflexivo, teórico-práctico y dinamizador los temas pertinentes para fomentar y desarrollar las competencias comunicativas en cuanto a la interpretación y producción de textos se refiere.

A razón de lo anterior, los docentes de la IEDA Nuestra Señora de las Mercedes, a través del proyecto Fortalecimiento de la Cultura Ciudadana y Democrática CT+I a 
través de la IEP apoyada en TIC en el Departamento de Magdalena, propuso a través de la implementación de las Tecnologías de la Información y Comunicación (TIC), en el contexto escolar, facilitar y fortalecer las capacidades de los estudiantes, en especial las correspondientes al área de lengua castellana, (interpretación y producción de textos); habilidades necesarias para el aprendizaje en todas las áreas del saber; así mismo, con el apoyo de estas herramientas el docente se valdrá de una gran diversidad de estrategias metodológicas innovadoras que le facilitaran el proceso de enseñanza, que lo convierte en un agente activo y facilitador del aprendizaje.

La interpretación y producción de textos a través de las TIC

Teniendo en cuenta que la finalidad de la investigación es necesario definir inicialmente el término de competencias, el cual según el Ministerio de Educación, es aquella destreza para enfrentarse a situaciones problemas en contextos académicos y sociales, respondiendo satisfactoriamente a este.

En este orden de ideas se entiende entonces por la interpretación y producción textual, como la capacidad de construir coherentemente una información, procesando y elaborando de manera clara las contenido del texto, relacionando dicho contenido con las conocimientos previos del lector, (Piacente y Tittarelli 2006), es fundamental en este sentido trabajar con herramientas y metodologías que apunten a potencializar dichas competencias, estando las primeras enfocadas en el análisis, reflexión y aprehensión de lo leído, mientras que las segundas se enfocan en la práctica, reglas de escritura como: fonética, ortografía, coherencia y la cohesión textual (Martos 1994).

Dado a todos los cambios que se han presentado en la práctica pedagógica por la incorporación de las tecnologías de la información y comunicación; es necesario incorporar estrategias didácticas, que permitan el desarrollo de competencias integrales del estudiante, donde se consoliden habilidades, destrezas y actitudes para la aplicación e intervención de herramientas tecnológicas en el ámbito pedagógico (Marín, Niebles, Sarmiento y Valvuena, 2015)

Respecto a eso Miranda, Muñoz, Porras y Ramírez (2006), propone que el uso de estas tas herramientas como estrategias pedagógicas, brindan un sin número de recursos como imágenes, videos, gráficos, y textos que facilitan y permiten para mejorar las capacidades de interpretación y producción textual, abriendo al estudiante la posibilidad de acceder a una ilimitada fuente de información para responder con un trabajo creativo y significativo.

Marqués (2006), por su parte considera que trabajar con las TIC en los procesos de aprendizaje no solo se apunta a fortalecer la escritura y producción de un texto, sí no que apunta a que el estudiante lograr generar un interacción entre, lo escrito y lo vivido, logrando fomentar el interés y la motivación de estos por la lectura y la escritura.

Otro aporte significativo es el que ofrece Toro (2017), quien manifiesta que las TIC como herramientas dinamizadora, logra facilitar los procesos interpretativos de textos en los estudiantes, logrando un proceso de interacción positivo entre docente y estudiante; generando cambios en la dinámica del proceso de enseñanza aprendizaje; brindándole al docente las herramientas para asumir un nuevo rol activo, dejando a un lado pedagogía tradicional de enseñanza, y diseñando actividades creativas y lúdica que le permitan al estudiante facilitar la adquisición de los saber y logren superar las dificultades en lectura y escritura, es necesario que los docentes como guías y orientadores del proceso, desarrollen la capacidad del estudiante de interpretar, argumentar, debatir, analizar, criticar y dar 
significado a toda información que se le sea suministrada. De ahí que los docentes se convierten en agentes de cambios, activos en un rol dinamizador que lleva al estudiante a descubrirse y ser autónomo en su propia búsqueda del saber. En el caso concreto de la mediación didáctica los recursos tecnológicos resultan de gran utilidad; al respecto Talavera y Marín (2015) destacan la necesidad de una concepción del trabajo docente innovadora, que coadyuve a la socialización de contenidos didácticos.

\section{Metodología}

La metodología de la investigación ayudará en el desarrollo de una revisión teórica de los elementos de integración curricular de las TIC desde los lineamientos que propone el programa ciclón, los cuales como herramienta de insumos se establecen desde el material pedagógico digital e impreso de las cartillas que ofrece el programa (Crissien, Reyes, Herrera, Herrera, Franco y Avendaño, s.f.).

Se asume un abordaje metodológica desde la investigación cualitativa, orientada desde la investigación acción, cuyo objetivo principal radica a en el cambio y mejora de una realidad educativa (Cerda 1997).

\section{Participantes}

La unidad de análisis estuvo constituida por 132 estudiantes de los grados tercero, cuarto, quinto y sexto de nivel primaria y secundaria de la Institución Educativa Departamental Agropecuaria "Nuestra Señora de las Mercedes" del corregimiento de Piñuela del Municipio de Pivijay Magdalena, distribuido de la siguiente manera: sede rural "El Amparo", ubicada en el corregimiento de Paraíso, del cuarto grado $4^{\circ}$ (30 estudiantes), del grado quinto $5^{\circ} \mathrm{A}(15$ estudiantes), quinto $5^{\circ} \mathrm{B}$ (16 estudiantes), sede "Bloque 2", ubicado en el corregimiento de Piñuela, estudiantes del cuarto $4^{\circ}(21$ estudiantes), quinto B $5^{\circ} 2$ (12 estudiantes) y sede principal de bachillerato grado sexto (26 estudiantes) equivalente al $8 \%$ de la población estudiantil total de la Institución, con edades que fluctúan entre los 9 y 15 años.

Técnicas e instrumentos de recolección de información

Respecto a las técnicas e instrumentos de recolección y organización de información, se tomó como fuente información la Revisión de los resultados de las pruebas saber pro 2016 para los grados de $3^{\circ} 5^{\circ}$ y $9^{\circ}$ en el área de lenguaje, se utilizó como técnica de recolección la observación participante, pues a través de esta en la cual el investigador, está en la capacidad de observar, escucha y participa del fenómeno estudiado en su contexto real (Hernández, Fernández y Baptista 2012); como instrumento de recolección se utilizó el diario de campo, donde se registraron las anotaciones más relevantes del proceso de observación. Para obtener dicha información se diseñaron serie de actividades teórico práctico, con el fin de observar el resultado de trabajar con las TIC, como estrategias para fortalecer las competencias de interpretación y producción de textos.

\section{Procedimiento}

Inicialmente se tomaron como fuente de información los resultados de las pruebas SABER 2016, con el fin de tener mayor claridad de las falencias específicas que presentan los estudiantes en cuanto a las competencias en la área de lenguaje, objetos de estudio de esta investigación. A partir de ese resultado se diseñaron las actividades, con el fin de impactar directamente en la problemática u objeto de estudio de la investigación; la observación de las actividades se realizó por cada grupo de trabajo en las sedes correspondientes; dichas actividades eran realizadas en la asignatura de lengua castellana, una vez por semana. 


\section{Resultados}

A continuación se presentan los resultados derivados de la realización de las acti- vidades, donde se implementaron las TIC, como la herramienta dinamizadora para fortalecer la interpretación y producción textual.

\section{TABLA 1}

Actividades para el fortalecimiento de la interpretación y producción de textos a través de las TIC.

\begin{tabular}{|c|c|c|}
\hline Actividad & Descripción de la actividad & Resultado \\
\hline $\begin{array}{l}\text { El libro } \\
\text { Viajero }\end{array}$ & $\begin{array}{l}\text { Se crearon con apoyo de las TIC, } \\
\text { relatos e historias escritos por los } \\
\text { estudiantes, que fueron depositados } \\
\text { en una maleta, la cual se trasladado } \\
\text { entre las distintas sedes y fueron } \\
\text { publicados en las redes sociales de la } \\
\text { escuela permitiendo la socialización } \\
\text { y el fomento de la creatividad en los } \\
\text { jóvenes }\end{array}$ & $\begin{array}{l}\text { A partir de la actividad realizada Se } \\
\text { halló que los estudiantes al saber que } \\
\text { sus relatos serian compartidos a través } \\
\text { de las redes sociales se esforzaron y } \\
\text { mostraron con mayor motivación para } \\
\text { trabajar, entregando trabajos con mayor } \\
\text { calidad, en menos tiempo del programado } \\
\text { y con buena argumentación y calidad } \\
\text { interpretativa }\end{array}$ \\
\hline $\begin{array}{l}\text { Mi Biblioteca en } \\
\text { Red }\end{array}$ & $\begin{array}{l}\text { En esta actividad se compartió con } \\
\text { los estudiantes a través de las redes } \\
\text { sociales diferentes tipos de cuentos y } \\
\text { lecturas para analizar y comprender } \\
\text { en familia. Se }\end{array}$ & $\begin{array}{l}\text { Los resultados de la actividad } \\
\text { permitieron identificar que los } \\
\text { estudiantes al trabajar de la mano } \\
\text { con las TIC, y apoyados por los } \\
\text { padres de familia, Mostraron en sus } \\
\text { interpretaciones mayor coherencia, } \\
\text { cohesión y organización. Así mismo se } \\
\text { generaron situaciones compartidas de } \\
\text { expresión de opiniones, sentimientos, } \\
\text { emociones, actividades que se les } \\
\text { dificultaba realizar con las tareas } \\
\text { anteriormente asignadas }\end{array}$ \\
\hline $\begin{array}{l}\text { El club de la } \\
\text { lectura. }\end{array}$ & $\begin{array}{l}\text { Se crearon espacios en la escuela } \\
\text { para la lectura, donde a través del } \\
\text { video beam con apoyo de imágenes se } \\
\text { proyectaron textos, los cuales debían } \\
\text { leer y luego interpretar. }\end{array}$ & $\begin{array}{l}\text { Los resultados arrojados en la actividad } \\
\text { fueron positivos, en la medida que lo } \\
\text { estudiantes participan de manera activa, } \\
\text { logrando generar un alto nivel de interés } \\
\text { por leer lo presentando, estos lograron } \\
\text { trabajar de manera participativa, con } \\
\text { mayores resultados, solicitando crear } \\
\text { más espacios para trabajar con la lectura. }\end{array}$ \\
\hline $\begin{array}{l}\text { Las TIC, y la } \\
\text { lectura. }\end{array}$ & $\begin{array}{l}\text { En esta actividad se asignaban } \\
\text { preguntas a los estudiantes, los } \\
\text { cuales debían consultarlas a través } \\
\text { de las WEB, interpretar el contenido } \\
\text { de la información, comprenderla para } \\
\text { luego producir un relato a partir de } \\
\text { lo investigado. }\end{array}$ & $\begin{array}{l}\text { Se halló que los estudiantes respondían } \\
\text { de manera más precisa y exacta las } \\
\text { preguntas, pues su nivel de análisis e } \\
\text { interpretación mostraba mejor calidad } \\
\text { que cuando se asignabas las actividades } \\
\text { por medio de elementos físicos como } \\
\text { libros o revistas. Posteriormente a } \\
\text { estas preguntas las evaluaciones que } \\
\text { se realizaron partir de ellas las notas } \\
\text { obtenidas por estos superaron el nivel } \\
\text { promedio que se manejaba en el aula de } \\
\text { clases. }\end{array}$ \\
\hline
\end{tabular}

Fuente. elaboración propia. 
En términos generales los resultaos de las actividades dejan evidencia que al trabajar con las TIC, los temas referentes a la interpretación y producción de textos, se genera un incremento significativo en los resultados académicos de los estudiantes; se logró despertar en ellos mayor nivel de motivación e interés, trabajando los temas de manera más amena, con un alto nivel de participación y producción.

Se percibió un alto nivel de interés a la hora de trabajar con imágenes, las redes sociales y búsquedas por la web, donde los resultados se reflejaron y al momento de realizar las actividades, donde entregaron las actividades escritas correctamente, con buen nivel analítico, crítico e interpretativo.

Así mismo, se logró una mejoría significativa en la comprensión y la redacción puesto que sus actividades fueron realizadas de manera clara, correcta y completa, despertándose en ellos el interés por seguir desarrollando actividades de lectura, y escritura.

La implementación de dichas actividades, mediadas por las TIC, mejoró notablemente la comprensión lectora y la producción de textos escritos en los estudiantes que participaron en el proyecto debido a que su promedio en el área de lengua castellana tuvo cambios positivos, incrementando su promedio académico.

\section{Discusión}

Mediante los resultados obtenidos se hace pertinente y necesario fortalecer las estrategias en pro del mejoramiento de la interpretación y producción de textos de los estudiantes de la Institución Educativa Departamental Agropecuaria Nuestra Señora De Las Mercedes, teniendo en cuenta los resultados derivados de las actividades realizadas, dejan en evidencia la importancia de trabajar con las TIC en el fortalecimiento de dichas competencias, pues de esta manera se logra impactar de forma significativa en el proceso de enseñanza-aprendizaje y por ende lograr obtener mejores resultados en las próximas pruebas a presentar en la institución ya sea a nivel externo e interno.

El desarrollo de proyectos de investigación como estrategia en el mecanismo de integración curricular de las TIC permite ser una alternativa de redefinición de las estrategias que adoptan los docentes para fortalecer su quehacer pedagógico. Los resultados de la experiencia de trabajo permitieron realizar un contraste en las competencias que poseían la población de estudiantes respecto al área de lenguaje antes de trabajar con las TIC y posterior a su implementación. Frente a los resultados obtenidos se considera que la aplicación de las distintas estrategias utilizadas como la del libro viajero, la maleta lectora, mi biblioteca en Red, permitieron fortalecer las habilidades en los estudiantes respecto a la interpretación y producción de texto.

Lo resultados expuesto manifiestan unaa adecuada integración de las TIC en el proceso de enseñanza aprendizaje, mediante el uso de diferentes programas y software, influiría significativamente en la adquisición de diversas competencias, útiles para la resolución de problemas, y en la comprensión y producción de textos.

Estos hallazgos también se relacionan con lo expuesto por Sáez (2011), quien plantea que trabajar con las TIC, en la ámbito de la lectura y escritura, facilita el desarrollo de estas competencias, pues a través de ella, los elementos que ofrecen las TIC, se desarrolla e incentiva la actividad por la lectura, fortaleciendo procesos cognitivos, y habilidades de expresión y creatividad, mejorando además las relaciones entre alumno y profesor logrando trabajar cooperativamente. 
Es necesario señalar que el uso de las TIC en el proceso de interpretación textual, motiva e incentiva a los estudiantes a enriquecer su aprendizaje, pues su uso como estrategia pedagógica permite ingresar a diversas información que facilita la adquisición, análisis e interpretación de textos a través de imágenes, videos y textos que generan concentración, mayor orientación y mejora la interpretación de los textos. Guzmán (2014).

A raíz de lo mencionado, es posible concluir que las TIC, contribuyen en gran medida al fortalecimiento de la interpretación y producción de textos en los estudiantes, por cuanto dinamizan las prácticas de aula y tienen en cuenta sus necesidades e interés. Partiendo de esto, se hace necesario a nivel institucional, lograr replantearse el ejercicio de la práctica pedagógica, actualizando e innovando el quehacer docente asumiendo las tecnologías de la información y comunicación como parte de su quehacer diario, dejando a atrás la practica pedagógica tradicional, para darle paso a una era de nuevas estrategias innovadoras que posibiliten mayores y mejores resultados.

\section{Referencias}

Barletta, N., Toloza, H., Villar, L., Rodríguez, A., Bovea, V. y Moreno, F. (2013). Enseñanza y aprendizaje de la lectura y la escritura: una confabulación en el contexto oficial. Lenguaje, 41(1). 133168.

Cerda, H. (1997). La investigación total. Bogotá, D.C.: Editorial Magisterio.

Crissien, T., Reyes, J., Herrera, B., Herrera, K., Franco, M., y Avendaño, I. Ruta (2016). Metodológica para introducir la investigación como estrategia pedagógica-IEP apoyada en TIC en el currículo. Recuperado de https://es.scribd.com/ document/327483212/CARTILLAApropiacion-Del-Programa-Ciclon-2.
Henao, C. (2016). Las TIC: estrategia para mejorar la competencia lectorainterpretativa en el área de lenguaje. Revista Universidad Católica Luis Amigó, 1. 126-148. https://doi. org/10.21501/25907565.2653

Henao, O. (2006). Evidencias de la investigación sobre el impacto de las tecnologías de información y comunicación en la enseñanza de la lectoescritura. Revista Educación y Pedagogía, XVIII. 44. 71-87.

Hernández, R., Fernández, C. y Baptista, P. (2012). Metodología de la investigación. México, D.F.: McGraw-Hill Education.

ICFES. (2017). Resultados de pruebas saber Pro. Recuperado de http:// www2.icfesinteractivo.gov.co/ReportesSaber359/consultaReporteEstablecimiento.jspx

Marin, F., Niebles, M., Sarmiento, M. y Valvuena, S. (2017). Mediación de las tecnologías de la información en la comprensión lectora para la resolución de problemas aritméticos de enunciado verbal. Revista Espacios, 38 (20).

Marqués, P. (2006). El papel de las TIC en el proceso de lectoescritura. México, D.F.: Editorial Planeta.

Martínez, O., Salgado, M., Cantillo, M. y Prada, E. (2015). Interpretando textos a través de las tic: Una propuesta pedagógica para los estudiantes de grado cuarto de básica primaria de la Institución educativa Olga González Arraut. [Tesis Doctoral]. Universidad de Cartagena, Cartagena.

Martos, F. (1994). La producción de texto: un método eficaz para reforzar competencias lingüísticas. En, Actas del Segundo Congreso Nacional de ASELE. Español para extranjeros: didáctica e investigación. (143-148). Madrid, España. 
Miranda, N., Muñoz, M., Porras, L. y Ramírez, D. (2006). Las TICS, nuevas alternativas en la superación de las dificultades en el aprendizaje escolar. [Tesis]. Universidad de Antioquia, Medellín.

Patiño, A. (2015). Tendencias tecnológicas que influyen en el aumento de la productividad empresarial. INGE CUC, 11(2), 84-96. https://doi.org/10.17981/ ingecuc.11.2.2015.09

Piacente, T. y Tittarelli, A. (2006). Comprensión y producción de textos en alumnos universitarios: la reformulación textual. Orientación y sociedad, 6 , 99-126.

Sáez, J. (2011) Utilización eficaz y actitudes que muestran los docentes en la aplicación de las tecnologías de la información y comunicación en educación primaria. [Tesis doctoral]. Universidad Nacional de Educación a Distancia, Madrid, España.
Spina, M., Rohvein, C., Urrutia, S., Roark, G., Paravié, D. y Corres, G. (2016). Aplicación del modelo SCOR en pymes metalmecánicas de Olavarría. INGE CUC, 12(2), 50-57. https://doi.org/10.17981/ ingecuc.12.2.2016.05

Talavera, R y Marín, F. (2015). Recursos tecnológicos e integración de las ciencias como herramienta didáctica. Revista de Ciencias Sociales RCS, 21(2), 337-346.

Villalobos, M., Gómez, M., y González, L. (2013). Promoción de la escritura creativa a través de talleres apoyados con tecnologías digitales en escuelas unidocentes. Revista Q, 8 (15), 1-19. 